\title{
Primeros resultados sobre la gestión del patrimonio natural. Diagnóstico de un Área Natural Protegida en Xalapa, Ver.
}

\author{
María de Lourdes Becerra Zavala1 - Omar González Rodríguez¹
}

\author{
Observatorio de Políticas Culturales
}

\begin{abstract}
RESUMEN
Se presentan algunos resultados correspondientes al Diagnóstico Sociocultural del Área Natural Protegida Parque Lineal Quetzalapan Sedeño (PLQS), cuyo propósito es proporcionar información que permita orientar y evaluar las estrategias comunicativas y de educación no formal en materia de patrimonio natural. En el mes de mayo de 2017 se aplicaron encuestas a adultos

e infantes en un radio de $2 \mathrm{~km}$ alrededor del PLQS, en vialidades urbanas con presencia de actividad económica y movilidad urbana significativa. En opinión de los infantes la basura es el problema más relevante alrededor de sus viviendas, en tanto que los adultos dividen su opinión entre falta de agua y delincuencia. Los adultos desconocen la existencia del PLQS aunque muestran disposición a colaborar en acciones a favor de su preservación. Aún falta aplicar más encuestas, pero ya se vislumbran indicadores sobre las posibilidades de la gestión cultural en materia de patrimonio natural.
\end{abstract}

\begin{abstract}
Some results are presented corresponding to the Sociocultural Diagnosis of the Protected Natural Area "Parque Lineal Quetzalapan Sedeño" (PLQS), whose purpose is to provide information to plan education (formal and non formal) and communication strategies in natural heritage. In May 2017, surveys were apply on adults and children about $2 \mathrm{~km}$ around PLQS, in urban mainstreets with significant economic activity and urban mobility. In the opinion of the infants, garbage is the most relevant problem around their homes, while adults divide their opinion between water shortage and delinquency. Adults are not aware of the existence of PLQS although they are willing to collaborate in actions in favor. There is still a need for more surveys, but indicators shows the possibilities of cultural management in terms of natural heritage.
\end{abstract}

Palabras Clave: patrimonio natural, representaciones sociales, gestión cultural, participación ciudadana.

Keywords: natural heritage, social representations, cultural management, citizen participation.

L a gestión cultural es parte del ejercicio de la ciudadanía. Es una modalidad de política en la que los ciudadanos, en términos de derechos y obligaciones, ejercen su poder para la solución o atención de necesidades de cualquier instancia, en colaboración para un bien comunitario. Es sinónimo de organización para la obtención de recursos y puesta en marcha de proyectos que beneficien de alguna manera directa o indirecta a sus pares.

El Observatorio de Políticas Culturales (OPC) de la Facultad de Antropología de la Universidad Veracruzana (FAUV) da seguimiento a las

1 Facultad de Antropología de la Universidad Veracruzana.lbecerra@uv.mx, el.guarache.indigente@gmail.com 
actividades de gestión y desarrollo cultural, a las prácticas y políticas culturales. El interés del OPC consiste en crear los medios para la reflexión y elaboración de políticas públicas y culturales, de acuerdo a las necesidades de las comunidades del estado de Veracruz.

El caso de Desarrollo Sustentable del Río Sedeño Lucas Martin A.C. es un ejemplo de la gestión cultural; su propósito es el de salvaguarda y divulgación del Área Natural Protegida Parque Lineal Quetzalapan-Sedeño (PLQS) y lleva cerca de 20 años de actividades de concientización ambiental, recuperación y acondicionamiento de áreas verdes, así como integración con la comunidad por medio de talleres ecológicos. Entre sus actividades de gestión cultural y de política pública están el nombramiento de un Área Natural Protegida, la instauración de una planta de tratamiento de aguas residuales en los límites municipales de Banderilla y Xalapa, y en el año 2015 lograron su Programa de Manejo del (ANP PLQS).

A partir de enero de 2017 el OPC estableció un acuerdo de colaboración, en el marco del
Convenio de Vinculación firmado en septiembre de 2016 por la UV, con la Asociación Civil Desarrollo Sustentable del Río Sedeño, Lucas Martin A.C., para medir el impacto de dicha Asociación. Para este año se desarrolla el Diagnóstico Sociocultural del PLQS con el propósito de trazar algunas propuestas de comunicación a las colonias aledañas, y así trazar una propuesta de gestión cultural del patrimonio natural que amplíe las actividades hasta ahora llevadas a cabo.

El OPC se ha dado a la tarea de construir indicadores, a través del método etnográfico, para una futura interpretación sociocultural del impacto que la A.C. ha tenido sobre los vecinos de los municipios de Xalapa y Banderilla, así como disposición a participar con la A.C.; para sugerir propuestas que fortalezcan las estrategias comunicativas de la Asociación y con ello su impacto social. Al inicio de nuestra investigación cualitativa se ha recurrido a técnicas como la georreferenciación, la observación, aplicación de encuestas.

\section{Ubicación del Río Sedeño en la Cuenca Hidrológica del Río Actopan, Veracruz}

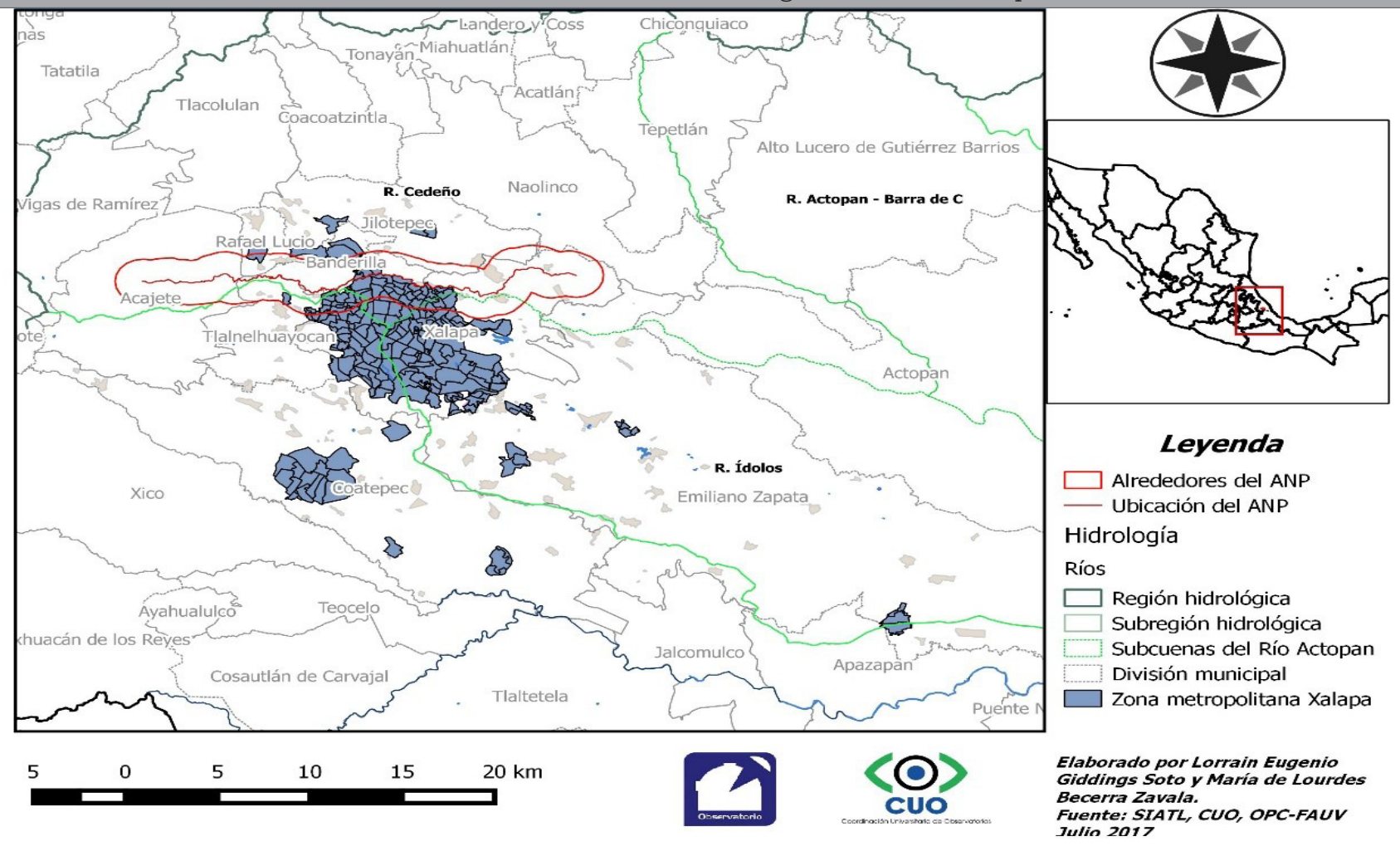

Figura 1. Mapa de localización del Área Natural Parque Lineal Quetzalapan Sedeño 
A continuación, se presentan algunos de los resultados de encuestas realizadas durante el mes de mayo del 2017. Aunque en las tablas se aprecien las zonas urbanas en las que se trabajó: avenidas de doble sentido con un gran número de comercios formales e informales con actividad entre semana y fines de semana, con paradas de autobuses a lo largo de ellas, y que forman parte de al menos 2 rutas de autobús urbano. Todos los datos corresponden a un análisis de Moda, excepto la edad.
Como se dijo anteriormente el OPC está encargado de generar las reflexiones y divulgar las actividades referentes a la gestión pública y las políticas culturales. Tan fundamental resulta el ejercicio de nuestros derechos como ciudadanos como nuestras obligaciones con el patrimonio natural; es nuestro deber como ciudadanos, y como especie, preservar el medio natural, no en vano nos va la supervivencia en ello.

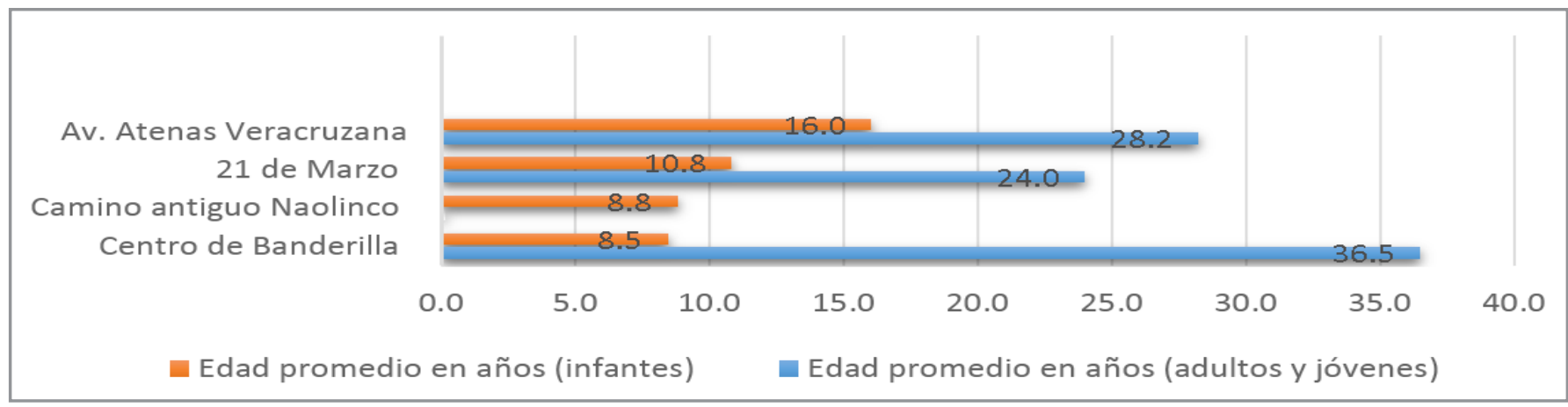

Gráfico 1. Edad promedio de los encuestados (mayo 2017). Fuente OPC-FAUV

Vialidades y opiniones de los encustados (adultos e infantes) (Mayo 2017)

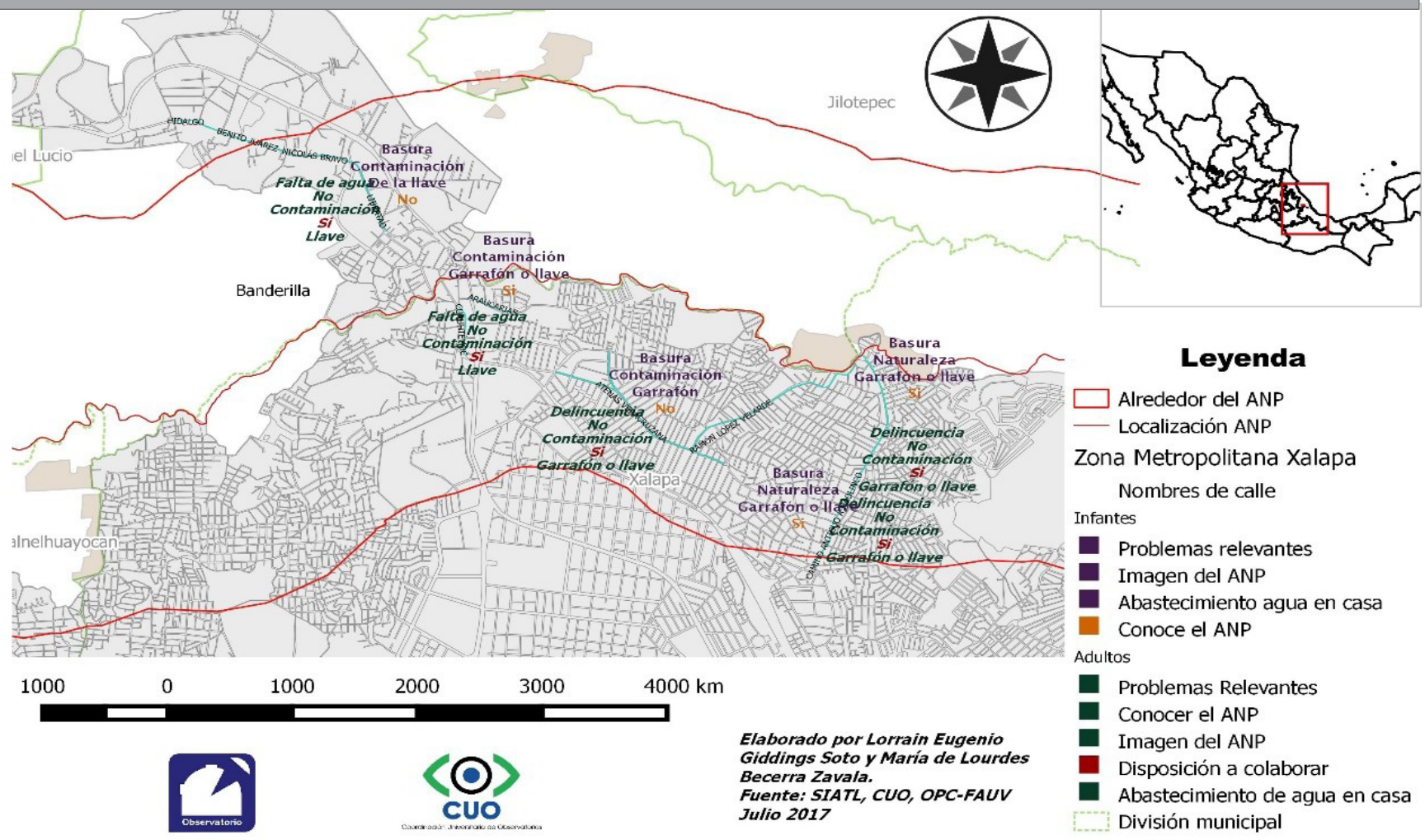

Gráfico 2. Opiniones sobre el ANP 